\title{
What's new in testosterone deficiency research?
}

\author{
Anil Kapoor, MD, FRCSC \\ Professor of Surgery (Urology), McMaster University, Associate Editor (Oncology), Canadian Urological Association Journal (CUAS), Hamilton, ON, Canada
}

Cite as: Can Urol Assoc J 2016;10(5-6Suppl2):S130-1. http://dx.doi.org/10.5489/cuai.3915

Testosterone replacement therapy may improve some aspects of cognitive ability and depression in men with testosterone deficiency syndrome.

Many of the symptoms of testosterone deficiency syndrome (TDS) - diminished muscle mass and strength, decreased bone mineral density, anorexia, decreased libido, fatigue, dysphoria, and irritability - overlap with symptoms of depressive illness. To evaluate the effect of testosterone replacement therapy (TRT) on cognitive function and depression in this population, researchers from South Korea conducted a prospective, placebo-controlled, single-blind trial of 128 men with TDS (calculated total testosterone less than $3.3 \mathrm{ng} / \mathrm{mL}$ with symptoms of hypogonadism); ${ }^{1} 64$ patients received $1000 \mathrm{mg}$ of testosterone undecanoate, while the other 62 men were counselled to modify their lifestyle. The group that received TRT experienced significant increases in serum testosterone levels, along with improved scores on the five-item International Index of Erectile Function (IIEF$5)$, the Beck's Depression Inventory (BDI), and the Aging Males' Symptoms (AMS) scale $(p<0.05)$. The group that received lifestyle counselling experienced improvements in IIEF-5 scores, but not in any other parameter. Neither group saw significant changes in the Korean Mini-Mental State Examination (K-MMSE). However, when results were limited to men with cognitive impairment (defined as a K-MMSE score of less than 25), K-MMSE scores improved significantly in the group that received TRT.

\section{Addition of TRT to a phosphodiesterase type 5 (PDE5) inhibitor is supe- rior to PDE5 inhibitor alone in improving lower urinary tract symptoms (LUTS) in men with low testosterone levels}

Tadalafil has shown efficacy in treating LUTS secondary to benign prostatic hyperplasia (BPH), as well as erectile dysfunction. Since testosterone regulates nitric oxide synthase and is necessary to achieve an optimum response to a PDE5 inhibitor, researchers in South Korea evaluated whether the addition of a transdermal gel containing testosterone $10 \mathrm{~g}$ would improve symptoms of LUTS in men with low testosterone levels undergoing tadalafil treatment compared with tadalafil alone. ${ }^{2}$ In their results presented at EAU 2016, IPSS improvements from baseline to 12 weeks were similar between the two groups. However, the group given tadalafil plus testosterone $(n=44)$ showed significant improvements from baseline in the International Prostate Symptom Score (IPSS) storage subscore, the IPSS-QOL score, and the IIEF-EF domain score compared with the men given tadalafil alone $(n=46)$. The addition of testosterone also resulted in significantly greater improvements in Global Assessment Question (GAQ) and Life Satisfaction Checklist (LSC) scores compared with tadalafil alone.

Testosterone replacement therapy does not appear to be associated with increased risk for prostate cancer or death due to cardiovascular disease

Recent clinical trial results have raised concerns about the oncological and cardiovascular safety of TRT. However, these clinical trials are generally conducted with highly selected patient populations. Finnish researchers evaluated the incidence of prostate cancer and cardiovascular mortality in men treated with TRT in the Finnish Prostate Cancer Screening Trial (FinRSPC), a population-based study in which the only exclusion criteria was a previous diagnosis of prostate cancer. ${ }^{3}$ The FinRSPC cohort was randomized between 1996 and 1999, with a median followup of 12 years. Of the 78615 men enrolled in FinRSPC, 776 (1\%) were treated with TRT. TRT use was not associated with an increased risk of either prostate cancer or cardiovascular disease, but was, in fact, associated with a significant decrease in the overall risk of prostate cancer (multivariable-adjusted HR 0.80; 95\% Cl 0.73-0.88), as well as non-significant decreases in Gleason 7-10 (HR 0.86; 95\% Cl 0.68-1.09) and metastatic prostate cancer (HR 0.56; 95\% Cl 0.25-1.26). 
Table 1. Risk of death due to cardiovascular disease among users and non-users of testosterone replacement therapy in the Finnish Prostate Cancer Screening Trial ${ }^{3}$

\begin{tabular}{|c|c|c|c|c|}
\hline & \multicolumn{4}{|c|}{ Cardiovascular disease death } \\
\hline & \multicolumn{2}{|c|}{ Primary cause } & \multicolumn{2}{|c|}{ Primary or contributory cause } \\
\hline & $\begin{array}{l}\text { HR (95\% Cl) } \\
\text { Age-adjusted }\end{array}$ & $\begin{array}{c}\text { HR (95\% Cl) } \\
\text { Multivariate-adjusted }\end{array}$ & $\begin{array}{c}\text { HR (95\% Cl) } \\
\text { Age-adjusted }\end{array}$ & $\begin{array}{c}\text { HR }(95 \% \mathrm{Cl}) \\
\text { Multivariate-adjusted }\end{array}$ \\
\hline Non-users & Reference & Reference & Reference & Reference \\
\hline Ever users & $0.70(0.50-0.91)$ & $0.75(0.58-0.97)$ & $0.76(0.61-0.95)$ & $0.81(0.65-1.00)$ \\
\hline \multicolumn{5}{|l|}{ Years of use } \\
\hline$\leq 1$ year & $0.63(0.44-0.91)$ & $0.66(0.46-0.96)$ & $0.72(0.54-0.97)$ & $0.76(0.56-1.02)$ \\
\hline $1-3$ years & $0.76(0.47-1.23)$ & $0.82(0.51-1.32)$ & $0.71(0.46-1.09)$ & $0.76(0.49-1.17)$ \\
\hline$>3$ years & $0.86(0.47-1.55)$ & $0.94(0.52-1.71)$ & 0.99 (0.61-1.59) & $1.08(0.67-1.74)$ \\
\hline
\end{tabular}

TRT was also associated with a reduction in cardiovascular disease mortality (HR 0.84; 95\% Cl 0.71-0.99) (Table 1). The greatest declines in risk were observed during the first year and were independent of the length of TRT use. A limitation of the study was that no information on testosterone levels was obtained. The discussion from the audience during this poster presentation was that these population-based data are important in reassuring physicians that TRT does not appear to be linked to an increase in the risk of prostate cancer or cardiovascular disease.

\section{References}

1. Shin HS, Park JS, Moon KH. The effect of testosterone replacement in men with testosterone deficiency syndrome on cognitive performance and depression. Eur Urol Suppl 2016;15(suppl 3):e1008. http:// dx.doi.org/10.1016/S1569-9056(16)61009-6

2. Park HJ, Park NC, Moon DG. Does concomitant testosterone replacement improve the response of tadalafil $5 \mathrm{mg}$ once daily in men with lower urinary tract symptoms? Eur Urol Suppl 2016;15 (suppl 3):e1013. http://dx.doi.org/10.1016/S1569-9056(16)61014-X

3. Murrola T, Rytkönen J, Talala K, et al. Prostate cancer incidence and cardiovascular mortality among users of testosterone replacement therapy in the Finnish Prostate Cancer Screening Trial. Eur Urol Suppl 2016;15(suppl 3):e776. http://dx.doi.org/10.1016/S1569-9056(16)60778-9 